\title{
Importancia de la actividad física sobre la inteligencia emocional y diferencias de género \\ Impact of physical activity on emotional intelligence and sex differences
}

Samuel Galdón López, Félix Zurita Ortega, Jose Luis Ubago Jiménez, Gabriel González Valero

Universidad de Granada (España)

Resumen. Los factores emocionales y su desarrollo en contextos donde abunda la práctica deportiva están en el punto de mira de estos últimos años. Esto es así pues la actividad física tiene una estrecha relación con la canalización de las emociones y su efecto en el rendimiento deportivo, así como las diferencias sexuales en la comprensión de estos términos. Por estas razones, la presente investigación presenta como principal objetivo describir el nivel de práctica de actividad física, inteligencia emocional y las diferencias entre sexos en escolares de educación primaria, con una muestra de 281 estudiantes de educación primaria de edades comprendidas entre 10 y 12 años en la provincia de Granada. Los instrumentos que se han utilizado fueron elTMMS-24, basado en Trait Meta-Mood Scale (TMMS) y un cuestionario ad-hoc para recoger las variables sociodemográficas. Los resultados muestran aquellos que practican más actividad física, siendo en su mayoría los chicos, tienen un mejor control de sus emociones en sus tres dimensiones, resaltando la capacidad de reparación, la cual presenta junto a la claridad emocional los valores más altos, donde las chicas presentan una leve mayoría. Además de diferencias significativas en las relaciones entre sexo-deportes y sexo-dimensiones de la inteligencia emocional.

Palabras clave: Inteligencia emocional; actividad física; deporte; escolares; educación física.

\begin{abstract}
Emotional elements have been the focus of attention in recent years in the context where sport practice is common. Therefore, physical activity has a close relationship with emotional expression and effect on sports performance, as well as sexual differences. Due to this fact, the main purpose of the present research is to describe the level of physical activity, emotional intelligence and sex differences in school children from primary education. A sample of 281 students aged between 10 and 12 years old in the city of Granada was studied. The instruments used are TMMS-24, based on Trait MetaMood Scale (TMMS) and an ad-hoc questionnaire to collect socio-demographic variables. The results indicate a higher level of physical activity, with most boys having better control of their emotions in the three dimensions, highlighting their ability to repair themselves, as well as their emotional clarity, with girls showing a slightly higher level. In addition, there are significant differences in the relationship between sex-sports and sex-dimensions of emotional intelligence.
\end{abstract}

Keywords: Emotional intelligence; physical activity; sport; schoolchildren; physical education.

\section{Introducción}

La mayoría de las personas dirán que la actividad física es algo gratificante, pues es importante estar físicamente activo y sentirse bien realizando, realizarla en sí está visto como una manera de ejecutar un gran esfuerzo cuya finalidad no es hacerlo de forma esporádica, sino que este se haga de manera habitual (Sachs et al., 2020). Así pues, la actividad física está presente en nuestro día a día y cada vez se tiene más conciencia de la importancia de la práctica de esta en nuestra vida diaria, pues la sociedad ha cambiado de tal forma que cada vez es menos saludable (Donnelly et al., 2017).

En este sentido, la Organización Mundial de la Salud expone una serie de recomendaciones para jóvenes que comprenden de los 5 a los 17 años realicen al menos 60

Fecha recepción: 12-12-20. Fecha de aceptación: 20-04-21

Samuel Galdón López

samuelgaldonlopez98@gmail.com minutos diarios de actividad física de una intensidad moderada a más enérgica. Si este tiempo mencionado es superado adquirirá un beneficio mayor para la salud, la actividad física convendría ser aeróbica y añadir, como mínimo tres veces por semana, actividades vigorosas que refuercen, en particular, los músculos y huesos (OMS, 2020). También, Sánchez et al. (2019) menciona algunos beneficios, entre ellos destaca que aquellos que practican actividad física desde la infancia y adolescencia, tienen mayor posibilidad de seguir haciendo en la edad adulta, siempre y cuando desde esas edades se motive y anime a hacerlo.

La práctica de actividad física es muy recomendada por varios autores por todos los beneficios que conlleva su realización. A este respecto, Warburton y Bredin (2017) señalan que realizar actividad física reduce el riesgo de mortalidad prematura, también señalan que los beneficios sólo pueden darse en un tiempo prolongado como realizar unos 150 minutos de menos a más intensidad y sobre todo promover estilos de vida salu- 
dable reduciendo el tiempo de sedentarismo, aumentando la actividad física diaria, mejorando la nutrición, dejando de tomar sustancias nocivas como alcohol o tabaco y manejando las situaciones de estrés.

La actividad física también está relacionada con la actividad mental y bienestar social, ya que aquellas personas que la realizan demuestran mejores destrezas psicológicas y disminuyen la ansiedad y el estrés (Acebes et al., 2019). Así pues, ésta puede definirse como una habilidad que tiene el ser humano para captar emociones, comprenderlas, expresarlas y tomar las medidas convenientes (Bueno, 2018). Asimismo, Acebes et al. (2019), la definen como una capacidad que tiene el ser humano para percibir con precisión, valorar y expresar emociones, una capacidad capaz de generar pensamientos, conocer, comprender y regular las emociones.

Por otra parte, la actividad física y el deporte ayuda a afrontar retos, colaborar formando parte de un equipo, así pues varios estudios señalan la relación que hay entre la inteligencia emocional y la actividad física como los trabajos de Crombie, Lombard y Noakes (2011) donde se analizaron a jugadores de criquet para ver su desarrollo de la inteligencia emocional donde vieron mejoras significativas en el desarrollo de la inteligencia emocional. El tener una buena inteligencia emocional ayuda a los jóvenes a conseguir sus metas con un mayor rendimiento como Valenzuela y Portillo (2018), donde en su estudio menciona que son los factores emocionales, sociales y afectivos los que juegan un papel importante desde la infancia, el cual hace que los niños y niñas se sientan motivados, con iniciativa y una mayor confianza. Así pues Mujica (2018) menciona que uno de los fines últimos de la Educación Física es que los escolares durante la realización de los diversos ejercicios y actividades construyan emociones positivas para su propio bienestar.

Este estudio tiene la finalidad de ver la relación que existe entre la actividad física, inteligencia emocional y sexo. En base a lo expuesto, este estudio persigue como principales objetivos de investigación: (a) Describir el nivel de práctica de actividad física, inteligencia emocional y sexo en escolares de educación primaria; (b) Establecer las relaciones entre la actividad física, inteligencia emocional y sexo en escolares de educación primaria.

\section{Material y Método}

\section{Participantes}

Este estudio de carácter descriptivo, relacional y de corte transversal, se realizó sobre una muestra de 281 alumnos de educación primaria, de entre 10 y 11 años de ambos sexos (49,8\% niños y 50,2\% niñas). La edad media total de la muestra empleada fue de $M=10,82$ años $(\mathrm{DT}=0,87)$. La obtención de esta muestra se realizó en diversos colegios de la provincia de Granada, mediante el muestreo intencional.

\section{Instrumentos}

Para el registro de los datos se utilizó un cuestionario ad-hoc para recoger las variables sociodemográficas, como el sexo, categorizada en hombre o mujer, la edad, centrada en alumnado de $5^{\circ}$ y $6^{\circ}$ de educación primaria. El tipo de deporte se categorizó atendiendo a la siguiente clasificación: ninguno, fútbol, baloncesto, danza, actividades en el medio natural, atletismo, senderismo, habilidades gimnásticas, actividades de raqueta, vóley, natación, rugby, ciclismo y otros.

Para el registro de la variable Inteligencia Emocional, se utilizó el TMMS-24, basado en Trait Meta-Mood Scale (TMMS), desarrollado por el grupo de investigación de Salovey, Mayer, Goldman,Turvey y Palfai (1995). La versión española utilizada en este estudio fue la de Fernández-Berrocal, Extremera y Ramos (2004). El TMMS-24 tiene tres dimensiones claves para la inteligencia emocional, con 8 ítems cada una de ellas. Este instrumento se compone de 24 ítems que evalúan la capacidad de atención, claridad y reparación de sus emociones, propias y ajenas, siendo los primeros del ítem 1 al 8, la segunda del ítem 9 al 16 y la tercera del ítem 17 al 24. Los ítems se evalúan mediante una escala Likert del 1 al 5, siendo $1=$ Nada de Acuerdo, 2=Algo de Acuerdo, 3=Bastante de Acuerdo, 4=Muy de Acuerdo y 5=Totalmente de acuerdo. Se realizó el análisis de fiabilidad de Alfa de Cronbach obteniendo á $=0,893$.

\section{Procedimiento}

En primer lugar, se dictaminó el número de centros educativos que había en la ciudad de Granada. El siguiente paso se centró en escribir una carta a la dirección de los correspondientes centros seleccionados donde se les pedía un permiso para poder pasar los test a los alumnos de quinto y sexto, para ellos se creó una carta dando información del estudio desde el departamento de Didáctica de la Expresión Musical, Plástica y Corporal de la Universidad de Granada. Una vez obtenida la aceptación por parte de los centros, se planteó a los colegios una fecha para la realización de los mismos, en horario lectivo, con el permiso añadido de los tutores de dichos cursos en los diferentes colegios. Se les infor- 
mó a los niños y niñas sobre el tema del estudio y sobre los datos que se iban a recoger, todos completamente anónimos. Tras la recogida de datos de los cuestionarios en papel, hubo que descartar 39 por hallarse incompletos o mal realizados, contando con una muestra final de 281 cuestionarios. Asimismo, se procedió a contrastar los datos con un software, IBM SPSS, para poder obtener los resultados. Finalmente, cabe destacar que esta investigación ha seguido las pautas de la declaración de Helsinki (1975) para investigaciones humanas y el permiso de la Universidad de Granada con el Comité de Ética 1230/CEIH/2020.

\section{Análisis de datos}

Para analizar los datos obtenidos en la investigación se utilizó el software estadístico IBM SPSS en su versión 25. Se utilizaron frecuencias para realizar el cálculo de los descriptivos básicos de las variables Sexo, Tipo de Deporte, Actividad Física practicada (AF), Federados, Inteligencia Emocional Atención (IEA), Inteligencia Emocional Claridad (IEC) e Inteligencia Emocional Reparación (IER), y de igual modo para la realización de un estudio relacional sobre la inteligencia emocional en función de la actividad física realizada por los estudiantes. Posteriormente se llevó a cabo un estudio relacional mediante tablas cruzadas, $\mathrm{T}$ de Student y ANOVA en función de las características de las variables.

\section{Resultados}

En cuanto a la variable tipo de deporte, se observa que un porcentaje mayor de estudiantes practican algún deporte, ya sea deportes de balón o deportes más artísticos, el más practicado es el fútbol con un $29,5 \%$ y un $14,6 \%$ aquellos que no practican ningún tipo de deporte. Una inmensa mayoría de los estudiantes practican actividad física, siendo un 85\%,4 aquellos que practican actividad física frente al 14,6\% que no practican en horario no lectivo.

Seguidamente, con respecto a la variable inteligencia emocional, tal y como se muestra en la tabla 1, la capacidad de atención evidencia como la mayoría, un $54,8 \%$, se encuentra en un valor medio de adecuada atención, seguidos de aquellos que tienen poca atención con un 31\% y como tercero y último, aquellos que tienen demasiada atención con un 14,2\% es decir, uno de cada diez alumnos presta mayor atención a las emociones y saben reconocer sus sentimientos. Respecto de la claridad, hay que destacar que una gran mayoría vuel- ven a mantenerse en la media un 55,9\%, presenta una claridad adecuada, pero casi un 30\% tendrían que mejorar su competencia para comprender las emociones y distinguirlas. Las personas que muestran excelente claridad, se presentan con el valor más bajo de todos, un $16 \%$, los que estarían en perfecta armonía, respecto a la atención, con sus emociones. En último lugar la reparación, entendida como capacidad para controlar emociones negativas y positivas, se observa que más de la mitad un $57,7 \%$, tienen una adecuada reparación, además de que un 23,8\% tienen un excelente control de emociones positivas y negativas, y un 18,5\% tienen que mejorar este control de las emociones.

Tabla 1 .

\begin{tabular}{|c|c|c|c|c|c|}
\hline \multicolumn{3}{|l|}{ Sexo } & \multicolumn{3}{|c|}{ IEA } \\
\hline & $\mathrm{N}$ & $\%$ & & $\mathrm{~N}$ & $\%$ \\
\hline Masculino & 140 & $49,8 \%$ & Poca Atención & 87 & $31,0 \%$ \\
\hline Femenino & 141 & $50,2 \%$ & Adecuada Atención & 154 & $54,8 \%$ \\
\hline \multicolumn{3}{|c|}{ Tipos de Deporte } & Demasiada Atención & 40 & $14,2 \%$ \\
\hline No practican & 41 & $14,6 \%$ & \multicolumn{3}{|c|}{ IEC } \\
\hline Fútbol & 83 & $29,5 \%$ & Debe mejorar & 79 & $28,1 \%$ \\
\hline Baloncesto & 26 & $9,3 \%$ & Adecuada & 157 & $55,9 \%$ \\
\hline Danza & 20 & $7,1 \%$ & Excelente & 45 & $16,0 \%$ \\
\hline M. Natural* & 7 & $2,5 \%$ & \multicolumn{3}{|c|}{ IER } \\
\hline Atletismo & 11 & $3,9 \%$ & Debe mejorar & 52 & $18,5 \%$ \\
\hline Senderismo & 9 & $3,2 \%$ & Adecuada & 162 & $57,7 \%$ \\
\hline Habilidades gimnásticas & 17 & $6,0 \%$ & Excelente & 67 & $23,8 \%$ \\
\hline Actividades de raqueta & 18 & $6,4 \%$ & \multicolumn{3}{|c|}{ Actividad Física } \\
\hline Voleibol & 5 & $1,8 \%$ & No & 41 & $14,6 \%$ \\
\hline Otros* & 21 & $7,5 \%$ & $\mathrm{Si}$ & 240 & $85,4 \%$ \\
\hline Natación & 15 & $5,3 \%$ & & & \\
\hline Ciclismo & 8 & $2,8 \%$ & & & \\
\hline
\end{tabular}

OSe hallan diferencias estadísticamente significativas $(p=, 000)$, propiciadas por aquellos que no practican deportes. Son en su mayoría niñas, con un 63,4\%, frente a los niños que se reduce a la mitad, un 36,6\%. Deportes de contacto como es el fútbol, es jugado en su inmensa mayoría por chicos con un 83\%, frente al 17\%, que supone las niñas. Sin embargo, aquellos que son de la rama más artística como las Habilidades Gimnasticas

\begin{tabular}{|c|c|c|c|c|c|c|}
\hline & & Masculino & Femenino & & Masculino & $\begin{array}{ll}\text { Femenino } & \text { Sig. }\end{array}$ \\
\hline \multirow{3}{*}{$\begin{array}{c}\text { No } \\
\text { practican }\end{array}$} & Recuento & 15 & 26 & \multirow{3}{*}{ H. Gimnásticas } & 1 & 16 \\
\hline & \% Deporte & $36,6 \%$ & $63,4 \%$ & & $5,9 \%$ & $94,1 \% \quad \mathrm{p}=, 000$ \\
\hline & $\%$ Sexo & $10,7 \%$ & $18,4 \%$ & & $0,7 \%$ & $11,3 \%$ \\
\hline \multirow{3}{*}{ Fútbol } & Recuento & 69 & 14 & \multirow{3}{*}{ Raqueta } & 8 & 10 \\
\hline & \% Deporte & $83,1 \%$ & $16,9 \%$ & & $44,4 \%$ & $55,6 \%$ \\
\hline & $\%$ Sexo & $49,3 \%$ & $9,9 \%$ & & $5,7 \%$ & $7,1 \%$ \\
\hline \multirow{3}{*}{ Baloncesto } & Recuento & 12 & 14 & \multirow{3}{*}{ Voleibol } & 0 & 5 \\
\hline & \% Deporte & $46,2 \%$ & $53,8 \%$ & & $0,0 \%$ & $100,0 \%$ \\
\hline & $\%$ Sexo & $8,6 \%$ & $9,9 \%$ & & $0,0 \%$ & $3,5 \%$ \\
\hline \multirow{3}{*}{ Danza } & Recuento & 4 & 16 & \multirow{3}{*}{ Otros } & 9 & 12 \\
\hline & \% Deporte & $20,0 \%$ & $80,0 \%$ & & $42,9 \%$ & $57,1 \%$ \\
\hline & $\%$ Sexo & $2,9 \%$ & $11,3 \%$ & & $6,4 \%$ & $8,5 \%$ \\
\hline \multirow{3}{*}{ A.M.N. } & Recuento & 2 & 5 & \multirow{3}{*}{ Natación } & 7 & 8 \\
\hline & $\%$ Deporte & $28,6 \%$ & $71,4 \%$ & & $46,7 \%$ & $53,3 \%$ \\
\hline & $\%$ Sexo & $1,4 \%$ & $3,5 \%$ & & $5,0 \%$ & $5,7 \%$ \\
\hline \multirow{3}{*}{ Atletismo } & Recuento & 8 & 3 & \multirow{3}{*}{ Ciclismo } & 4 & 4 \\
\hline & $\%$ Deporte & $72,7 \%$ & $27,3 \%$ & & $50,0 \%$ & $50,0 \%$ \\
\hline & $\%$ Sexo & $5,7 \%$ & $2,1 \%$ & & $2,9 \%$ & $2,8 \%$ \\
\hline \multirow{3}{*}{ Senderismo } & Recuento & 1 & 8 & & & \\
\hline & $\%$ Deporte & $11,1 \%$ & $88,9 \%$ & & & \\
\hline & \% Sexo & $0,7 \%$ & $5,7 \%$ & & & \\
\hline
\end{tabular}


(HG) y danza, en su mayoría son chicas, con un 94\% y $80 \%$ respectivamente, frente al $6 \%$ y el $20 \%$ de los chicos.

\begin{tabular}{|c|c|c|c|c|c|c|}
\hline Sexo & & Poca Atención & $\begin{array}{l}\text { Adecuada } \\
\text { Atención }\end{array}$ & $\begin{array}{c}\text { Demasiada } \\
\text { Atención }\end{array}$ & Total & Sig. \\
\hline \multirow{3}{*}{ Masculino } & Recuento & 41 & 82 & 17 & 140 & \multirow{3}{*}{$\mathrm{p}=0,400$} \\
\hline & \% Sexo & $29,3 \%$ & $58,6 \%$ & $12,1 \%$ & $100,0 \%$ & \\
\hline & $\%$ IEA & $47,1 \%$ & $53,2 \%$ & $42,5 \%$ & $49,8 \%$ & \\
\hline \multirow{3}{*}{ Femenino } & Recuento & 46 & 72 & 23 & 141 & \\
\hline & \% Sexo & $32,6 \%$ & $51,1 \%$ & $16,3 \%$ & $100,0 \%$ & \\
\hline & $\%$ IEA & $52,9 \%$ & $46,8 \%$ & $57,5 \%$ & $50,2 \%$ & \\
\hline \multirow{4}{*}{ Total } & Recuento & 87 & 154 & 40 & 281 & \\
\hline & $\%$ Sexo & $31,0 \%$ & $54,8 \%$ & $14,2 \%$ & $100,0 \%$ & \\
\hline & $\%$ IEA & $100,0 \%$ & $100,0 \%$ & $100,0 \%$ & $100,0 \%$ & \\
\hline & & Debe mejorar & Adecuada & Excelente & Total & Sig. \\
\hline \multirow{3}{*}{ Masculino } & Recuento & 38 & 78 & 24 & 140 & \multirow{3}{*}{$\mathrm{p}=0,854$} \\
\hline & $\%$ Sexo & $27,1 \%$ & $55,7 \%$ & $17,1 \%$ & $100,0 \%$ & \\
\hline & $\% \operatorname{IEC}$ & $48,1 \%$ & $49,7 \%$ & $53,3 \%$ & $49,8 \%$ & \\
\hline \multirow[b]{3}{*}{ Femenino } & Recuento & 41 & 79 & 21 & 141 & \\
\hline & $\%$ Sexo & $29,1 \%$ & $56,0 \%$ & $14,9 \%$ & $100,0 \%$ & \\
\hline & $\% \operatorname{IEC}$ & $51,9 \%$ & $50,3 \%$ & $46,7 \%$ & $50,2 \%$ & \\
\hline \multirow{3}{*}{ Total } & Recuento & 79 & 157 & 45 & 281 & \\
\hline & $\%$ Sexo & $28,1 \%$ & $55,9 \%$ & $16,0 \%$ & $100,0 \%$ & \\
\hline & $\% \operatorname{IEC}$ & $100,0 \%$ & $100,0 \%$ & $100,0 \%$ & $100,0 \%$ & \\
\hline
\end{tabular}

Se han encontrado diferencias estadísticamente significativas $(p=, 036)$, propiciadas porque aquellos estudiantes que no practican deporte, siendo estos un 31,7\%, prestan poca atención emocional, un 15\%, frente a aquellos que presentan una adecuada atención emocional, un $53,7 \%$, son en su mayoría estudiantes que realizan deporte, destacando las actividades acuáticas como natación $(73,3 \%)$ y practican deportes artísticos con un $64,7 \%$, en el caso de habilidades gimnásticas y un 50\% en la danza y aquellos que juegan a deportes de raque-

\begin{tabular}{|c|c|c|c|c|c|}
\hline \multicolumn{6}{|c|}{ ticados y Atención Emocional } \\
\hline & & Poca Atención & Adecuada & Demasiada & Sig. \\
\hline & & & Atención & Atención & \\
\hline \multirow{3}{*}{ No practican } & Recuento & 13 & 22 & 6 & \multirow{3}{*}{$\mathrm{p}=0,036$} \\
\hline & $\%$ Deporte & $31,7 \%$ & $53,7 \%$ & $14,6 \%$ & \\
\hline & $\%$ IEA & $14,9 \%$ & $14,3 \%$ & $15,0 \%$ & \\
\hline \multirow{3}{*}{ Fútbol } & Recuento & 22 & 49 & 12 & \multirow{3}{*}{$\mathrm{p}=0,036$} \\
\hline & $\%$ Deporte & $26,5 \%$ & $59,0 \%$ & $14,5 \%$ & \\
\hline & $\%$ IEA & $25,3 \%$ & $31,8 \%$ & $30,0 \%$ & \\
\hline \multirow{3}{*}{ Baloncesto } & Recuento & 9 & 13 & 4 & \multirow{3}{*}{$\mathrm{p}=0,036$} \\
\hline & $\%$ Deporte & $34,6 \%$ & $50,0 \%$ & $15,4 \%$ & \\
\hline & $\%$ IEA & $10,3 \%$ & $8,4 \%$ & $10,0 \%$ & \\
\hline \multirow{3}{*}{ Danza } & Recuento & 9 & 10 & 1 & \multirow{3}{*}{$\mathrm{p}=0,036$} \\
\hline & $\%$ Deporte & $45,0 \%$ & $50,0 \%$ & $5,0 \%$ & \\
\hline & $\%$ IEA & $10,3 \%$ & $6,5 \%$ & $2,5 \%$ & \\
\hline \multirow{3}{*}{ A.M.N. } & Recuento & 2 & 4 & 1 & \multirow{3}{*}{$\mathrm{p}=0,036$} \\
\hline & $\%$ Deporte & $28,6 \%$ & $57,1 \%$ & $14,3 \%$ & \\
\hline & $\%$ IEA & $2,3 \%$ & $2,6 \%$ & $2,5 \%$ & \\
\hline \multirow{3}{*}{ Atletismo } & Recuento & 2 & 7 & 2 & \multirow{3}{*}{$\mathrm{p}=0,036$} \\
\hline & $\%$ Deporte & $18,2 \%$ & $63,6 \%$ & $18,2 \%$ & \\
\hline & \% IEA & $2,3 \%$ & $4,5 \%$ & $5,0 \%$ & \\
\hline \multirow{3}{*}{ Senderismo } & Recuento & 3 & 5 & 1 & \multirow{3}{*}{$\mathrm{p}=0,036$} \\
\hline & $\%$ Deporte & $33,3 \%$ & $55,6 \%$ & $11,1 \%$ & \\
\hline & $\%$ IEA & $3,4 \%$ & $3,2 \%$ & $2,5 \%$ & \\
\hline \multirow{3}{*}{ H. Gimnásticas } & Recuento & 4 & 11 & 2 & \multirow{3}{*}{$\mathrm{p}=0,036$} \\
\hline & $\%$ Deporte & $23,5 \%$ & $64,7 \%$ & $11,8 \%$ & \\
\hline & $\%$ IEA & $4,6 \%$ & $7,1 \%$ & $5,0 \%$ & \\
\hline \multirow{3}{*}{ Raqueta } & Recuento & 2 & 14 & 2 & \multirow{3}{*}{$\mathrm{p}=0,036$} \\
\hline & $\%$ Deporte & $11,1 \%$ & $77,8 \%$ & $11,1 \%$ & \\
\hline & $\%$ IEA & $2,3 \%$ & $9,1 \%$ & $5,0 \%$ & \\
\hline \multirow{3}{*}{ Voleibol } & Recuento & 3 & 0 & 2 & \multirow{3}{*}{$\mathrm{p}=0,036$} \\
\hline & $\%$ Deporte & $60,0 \%$ & $0,0 \%$ & $40,0 \%$ & \\
\hline & $\% \mathrm{IEA}$ & $3,4 \%$ & $0,0 \%$ & $5,0 \%$ & \\
\hline \multirow{3}{*}{ Otros } & Recuento & 12 & 8 & 1 & \multirow{3}{*}{$\mathrm{p}=0,036$} \\
\hline & $\%$ Deporte & $57,1 \%$ & $38,1 \%$ & $4,8 \%$ & \\
\hline & $\%$ IEA & $13,8 \%$ & $5,2 \%$ & $2,5 \%$ & \\
\hline \multirow{3}{*}{ Natación } & Recuento & 2 & 11 & 2 & \multirow{3}{*}{$\mathrm{p}=0,036$} \\
\hline & $\%$ Deporte & $13,3 \%$ & $73,3 \%$ & $13,3 \%$ & \\
\hline & $\% \mathrm{IEA}$ & $2,3 \%$ & $7,1 \%$ & $5,0 \%$ & \\
\hline & Recuento & 4 & 0 & 4 & \\
\hline Ciclismo & $\%$ Deporte & $50,0 \%$ & $0,0 \%$ & $50,0 \%$ & $\mathrm{p}=0,036$ \\
\hline & $\%$ IEA & $4,6 \%$ & $0,0 \%$ & $10,0 \%$ & \\
\hline
\end{tabular}

tas, siendo la más alta con un 77,8\%. Finalmente, los que muestran demasiada atención, el grupo menos numeroso con un $14,6 \%$, son aquellos que realizan ciclismo con un $50 \%$ y atletismo con un $18,2 \%$.

\section{Discusión y conclusiones}

En este estudio se ha observado que en la variable deportes practicados, un porcentaje mayor de estudiantes lo realizan, siendo el que más el fútbol, en su contra, el menos practicado es el voleibol. Una inmensa mayoría de los estudiantes practican actividad física, frente a la pequeña parte que no realizan ningún tipo de actividad física, datos muy similares se encuentran en los estudios de Dudley, Okely, Pearson y Cotton (2011) afirmando que aquellos que practican actividades más animadas y que requieran una mayor implicación en las clases de Educación Física.

Además, las diferencias que se han encontrado por sexo, respecto a los deportes practicados, evidencian los deportes de colaboración y oposición, como es el fútbol, son mayoritariamente practicados por niños, y aquellas actividades más recreativas son preferidas por las niñas como indica en otro estudio Marambio et al. (2020) que señala valores similares a la muestra dada en el presente estudio, pues los niños y las niñas practican el deporte suficiente en horario extracurricular, con la curiosidad de que las niñas tienen más actividad que los niños. En contraposición, Greca, Silva y Loch (2016) obtuvieron datos similares donde la prevalencia de la inactividad física fue mayor en las niñas que en los niños. Esto puede deberse porque en la adolescencia suelen ser las niñas las que tienden a abandonar la práctica de actividades físico-deportivas como en estudios de Meneses y Ruiz (2017).

En cuanto a las cifras sobre actividad física realizada por los estudiantes, se ve como la gran mayoría, la practican en horario extracurricular, a este respecto Kahan y McKenzie (2019) defienden que para promover la práctica de actividad física en horario extracurricular es necesario tener unos buenos medios de comunicación y con ello ofertar las diferentes actividades más adecuadamente. También, se puede ver como visiblemente un mayor número de estudiantes tienen un modo de vida activo, ya que unos tres cuartos de la muestra practican entre dos y tres días en semana (Chacón et al., 2016). Datos muy relevantes dados por Greca et al. (2016) señalan que uno de los motivos por los cuales los niños en edad escolar y adolescencia practican menos actividad física, es que los padres suelen asociar realizar de- 
porte con un rendimiento bajo en los estudios.

Posteriormente, sobre la capacidad de atención, se evidencia como la mayoría se encuentra en el valor de adecuada atención, seguidos de aquellos que tienen poca atención y finalmente, aquellos que tienen demasiada atención. El menor dato se encuentra en aquellos que presentan demasiada atención y un tercio de la muestra presenta poca atención emocional, datos muy similares obtuvo Santos (2017), en el cual los niños en Educación Primaria suelen tener mejores resultados en atención, pues se centra en el apoyo familiar para aumentar los niveles de atención en los niños, siendo una de las capacidades esenciales pata simpatizar con las demás personas.

Asimismo, Estrada et al., (2020) obtuvieron datos similares a la presente investigación, muestran que una atención alta puede deberse al clima de aula, pues no solo la familia influye en las emociones del alumnado, ya que, se permanecen durante más tiempo en las aulas que en casa, sustentada esta hipótesis por Escalante (2017). Respecto de la claridad, hay que destacar que una gran mayoría vuelven a mantenerse en la media, presentan una claridad adecuada, pero casi un tercio tendrían que mejorar su competencia para comprender las emociones y distinguirlas. Las personas que muestran excelente claridad, se presentan con el valor más bajo de todos, los que estarían en perfecta armonía, respecto a la atención, con sus emociones.

Además, en esta se presentan valores similares a la atención emocional, siendo más de la mitad aquellos que se encuentran en valores medios, una pequeña parte de la muestra son la que presenta una excelente claridad y casi el tercio de los participantes tienen que mejorar, así mismo Estrada et al., (2020), afirma estos datos pues señala que aquellas personas que son emocionalmente inteligentes son capaces de reconocer y expresar sus emociones. Esto supone que la mayor parte de los participantes tendrán en un futuro más posibilidad de tener un control de sus emociones, al mismo tiempo de tener mayor éxito en la resolución de problemas.

En último lugar, respecto a la reparación, se observa que más de la mitad tienen una adecuada reparación, además de que un quinto tienen un excelente control de emociones positivas y negativas, y una menor parte tiene que mejorar este control de las emociones, se evidencia una gran parte niveles adecuados de reparación, por otra parte, aquellos que presentan unos niveles excelentes son el veinticuatro por ciento, para finalizar, una menor parte de la muestra presenta tiene que mejorar en reparación emocional. Estos datos señalan que los estudiantes tienen un buen control de sus emociones positivas y negativas. En otro estudio Fernández y Martín (2015), se indica que tener buenos niveles de inteligencia emocional mejora mejoran en la salud física, bienestar, autocontrol y sociabilidad.

En cuanto a los deportes de contacto como es el fútbol, es jugado en su inmensa mayoría por chicos, frente a las niñas. Sin embargo, aquellos deportes de la rama artística como las habilidades gimnasticas (HG) y danza, en su mayoría son practicados por chicas. Esto se ha debido a que los niños y niñas que practican deporte tienen una mejor gestión de sus emociones, estudios de Singh (2017) y Vaquero et al., (2020) coinciden que la actividad física se relaciona de manera significativa con la inteligencia emocional y subescalas, como la autoconciencia, estabilidad emocional, manejo de relaciones, integridad y autodesarrollo. En cuanto a las diferencias halladas en la variable sexo y tipo de deportes se debe a los cambios que hay a nivel madurativo entre los hombres y las mujeres, cabios hormonales y por recibir más o menos apoyo en las familias, como en estudios de Marambio et al., (2020) y Meneses y Ruiz (2017). Además, se han hallado diferencias estadísticamente significativas, propiciadas porque aquellos que no practican deportes son en su pluralidad niñas, frente a los niños que se reduce a la mitad. Esto puede deberse a lo que ya he explicado en anteriores puntos, donde las niñas en la preadolescencia y adolescencia tienen una mayor tasa de abandono en actividades físico-deportivas que los niños. Laird, Fawkner y Niven (2018) y Peral et al., (2020) obtuvieron en su informe que puede darse por recibir más o menos apoyo de las familias.

Del Rosal et al., (2016) señalan que los niños y niñas muestran valores adecuados en los componentes de la inteligencia emocional. Se han observado datos similares en cuanto a inteligencia emocional y sus diferencias de género, pues son las chicas quien obtiene mejores puntuaciones en atención emocional, aunque no son estadísticamente significativas. También Martínez (2018), en sus análisis de la competencia emocional señala que son las niñas las que gestionan ligeramente mejor sus emociones, frente a los niños, datos muy similares al estudio de Acebes et al., (2019). También, Doyle yThompson (2020) plantean en su estudio sobre los estereotipos e inteligencia emocional, mejores resultados en los hombres, aunque plantean que estos datos pueden haber sido afectados por los estereotipos de género. Consecutivamente, se observaron diferencias, 
propiciadas por aquellos estudiantes que no practican deporte, siendo estos la menor parte de los que prestan poca atención emocional, frente a aquellos que presentan una adecuada atención emocional que realizan deporte, destacando las actividades acuáticas como natación y practican deportes artísticos. Finalmente, los que muestran demasiada atención, el grupo menos numeroso. Sáenz (2019), reafirma que la práctica de actividad física fortalece las emociones positivas y evita aquellas que son negativas, además sostiene que tener una buena inteligencia emocional mejora la eficacia deportiva.

Para finalizar, se ha observado que, al relacionar el sexo y la actividad física, no se encuentran diferencias estadísticamente significativas, aunque se aprecian valores ligeramente superiores en varones. En un estudio realizado por Richard et al., (2020), se encontraron datos similares, donde los niños tenían mejor forma física porque practicaban más deporte. Por un lado, con la relación entre los tres niveles de inteligencia emocional y el sexo, no se han encontrado diferencias estadísticamente significativas, pero tienden los valores a ser superiores en chicas, más que en chicos, datos similares se reportan en el estudio de Venkatappa et al., (2012). En cuanto a la relación entre los niveles de inteligencia emocional y la práctica de actividad física, se observaron diferencias estadísticamente significativas en la atención, explicadas anteriormente, sin embargo, en la claridad y la reparación no se han encontrado estas. Finalmente, la relación dada entre los tres niveles de inteligencia emocional y actividad física muestran que no se han divisado diferencias significativas en ninguno de ellos.

En cuanto a las limitaciones, la primera que se ha encontrado es el estudio descriptivo de corte transversal, no permite estables relaciones causales entre variables, así como el cálculo real pues los datos de la muestra se han centrado en una determinada población, espacio y tiempo, por lo que otra limitación dada por esto es que, al darse a una determinada población, no se ha transportado a nivel nacional.

Teniendo en cuenta los objetivos de esta investigación describir el nivel de práctica de actividad física, inteligencia emocional y sexo en escolares de educación primaria y establecer las relaciones entre la actividad física, inteligencia emocional y sexo en escolares de educación primaria, se plantean las siguientes conclusiones: los estudiantes presentan valores elevados de actividad física. También se ha observado que en las tres dimensiones de la inteligencia emocional presentan niveles adecuados, resaltando la capacidad de reparación, la cual presenta junto a la claridad emocional los valores más altos y en último lugar, la atención emocional con valores más bajos.

Respecto a la relación entre sexo y actividad física hay que destacar que los chicos tienden a realizar más actividad física en horario extraescolar que las chicas. Seguidamente, en la relación entre actividad física e inteligencia emocional, en sus tres dimensiones, destacan aquellas personas que presentan valores más altos en inteligencia emocional, influenciadas por la práctica de esta. Aunque cuando se han comparado resultados entre sexo, las chicas presentan mejores resultados.

\section{Referencias}

Acebes, S., Diez,V., $\quad$ Esteban, G., y Rodríguez, R. (2019). Physical activity and emotional intelligence among undergraduate students: A correlational study. BMC Public Health, 19(1), 12-41. https://doi.org/10.1186/s12889019-7576-5

Bueno, S. (2018). La educación emocional a través de la música en educación infantil.La empatía. (Trabajo de fin de grado). Universidad de Valladolid, Valladolid.

Chacón, R.,Arufe,V., Cachón, J.,Zagalaz, M.,y Castro, D. (2016). Estudio relacional de la práctica deportiva en escolares según el género. SporTK: Revista Euroamericana de Ciencias del Deporte, 5(1), 85-92. https://doi.org/10.6018/249161

Crombie, D., Lombard, C., y Noakes, T. (2011). Increasing Emotional Intelligence in Cricketers: An Intervention Study. International Journal of Sports Science y Coaching, 6(1), 69-86. https://doi.org/10.1260/1747-9541.6.1.69

Del Rosal, I., Dávila, Mª A., Sánchez, S., y Bermejo, M. L. (2016). La inteligencia emocional en estudiantes universitarios: Diferencias entre el grado de maestro en educación primaria y los grados en ciencias. INFAD. Revista de psicología, 2(1), 51-61. https://doi.org/10.17060/ijodaep.2016.n1.v2.176

Donnelly, E., Hillman, H., Greene, L., Hansen, M., Gibson, A., Sullivan, K., Poggio, J., Mayo, S., Lambourne, K., Szabo-Reed, N., Herrmann, D. Honas, J. Scudder, R., Betts, L., Henley, K., Hunt, L., yWashburna,A. (2017). Physical activity and academic achievement across the curriculum: Results from a 3-year clusterrandomized trial. Preventive Medicine, 99,140-145. https:// doi.org/10.1016/j.ypmed.2017.02.006

Doyle, A., y Thompson, E. (2020). La influencia de la amenaza estereotipada y las teorías implícitas de la emoción en las diferencias de género en la inteligencia emocional. The Journal of Men's Studies, 1-25.

Dudley, D., Okely,A., Pearson, P.y Cotton,W. (2011).A systematic review of the effectiveness of physical education and school sport interventions targeting physical activity, movement skills and enjoyment of physical activity. European Physical Education Review, 17(3), 352-378. https://doi.org/10.1177/ $1356336 X 11416734$ 
Escalante, M. (2017). Inteligencia emocional y clima social escolar de los estudiantes del VII ciclo de la institución educativa «Los Libertadores», San Martin de Porres, Lima - 2015. Tesis doctoral, Universidad CésarVallejo.

Estrada,E., DelaTorre, M., Mamani,H., y Zuloaga, M.(2020). La inteligencia emocional y el clima de aula en estudiantes de educación superior. SCIÉNDO, 23(1), 53-58. http:// dx.doi.org/10.17268/sciendo.2020.008

Fernández,E. G., y Martín, M.D. (2015). Dimensions of emotional intelligence related to physical and mental health and to health behaviors. Frontiers in Psychology, 6,317. https://doi.org/ 10.3389/fpsyg. 2015.00317

Fernández-Berrocal, P., Extremera, N., y Ramos, N. (2004). Validity and reliability of the Spanishmodified version of the Trait Meta-Mood Scale.Psychological Reports, (94), 751 755. https://doi.org/10.2466/pr0.94.3.751-755

Greca, J., Silva, D. y Loch, M. (2016). Physical activity and screen time in children and adolescents in a medium size town in the South of Brazil. Revista Paulista de Pediatría, 34(3), 316-322. http:/ /dx.doi.org/10.1016/j.rppede.2016.01.001

Kahan, D., y McKenzie, T. (2019). School Websites: A Physical Education and Physical Activity Content Analysis. Journal of School Health, 90(1),47-56. https://doi.org/10.1111/ josh.12851

Laird,Y., Fawkner, S., y Niven, A. (2018). A grounded theory of how social support influences physical activity in adolescent girls. International Journal of Qualitative Studies on Health and Well-being, 13(1), 1435099. https://doi.org/10.1080/ 17482631.2018.1435099

Marambio, M., Núñez, T., Ramírez, J., Ramírez, P., Palma, X., y Rodríguez, F. (2020). Relación entre la actividad física escolar y extraescolar en estudiantes de escuelas públicas chilenas. Retos. Nuevas tendencias en Educación Física, Deporte y Recreación. 37, 393-399. https://doi.org/10.47197/ retos.v37i37.72851

Martínez, A. (2018). Competencias emocionales y rendimiento académico en los estudiantes de Educación Primaria. Psychology, Society, y Education, 11(1), 15-25.

Meneses, M., y Ruiz, J. (2017). Estudio longitudinal de los comportamientos y el nivel de actividad físico-deportiva en el tiempo libre en estudiantes de Costa Rica, México y España. Retos: nuevas tendencias en educación fisica, deporte y recreación, 31, 219226. https://doi.org/10.47197/retos.v0i31.53396

Mujica, F. (2018). Las emociones en la educación física escolar. El aporte de la evaluación cualitativa. EmásF. Revista Digital de Educación Física, 51,64-78

Organización Mundial de la Salud. (2020). Estrategia mundial sobre régimen alimentario, actividad fisica y salud. Actividad fisica. https: / /www.who.int/dietphysicalactivity/pa/es/

Peral, Á., Cuadrado, E., Perea, J., Navia, B., López,A., y Ortega, R. (2020). Physical activity practice and sports preferences in a group of Spanish schoolchildren depending on sex and parental care:A gender perspective. BMC Pediatrics, 20(1), 337. https: / /doi.org/10.1186/s12887-020-02229-Z
Richard, G., Mekoulou, N., Assomo-Ndemba, P., Hamadou, A., Biassi, O., Tsobgny-Panka, C., Mbah, G., Honoré, M, S. y Temfemo, A. (2020). Gender Differences in Physical Fitness among Cameroonian School Children Aged 10 to 15 Years in Yaounde City. International Journal of School Health, 7(3), 45-54. https://doi.org/10.30476/intjsh.2020.86621.1086

Sachs, L., Tashman, S., y Razon, S. (2020). Performance Excellence: Stories of Success from the RealWorld of Sport and Exercise Psychology. Philadelphia: Rowman y Littlefield.

Sáenz, P. (2019). Las emociones: necesidad de su programación para una actividad más saludable. E-Motion. Revista de Educación, Motricidad e Investigación, 13, 59-81. https://doi.org/ 10.33776/remo.v0i13.3801

Salovey, P., Mayer, J., Goldman, S., Turvey, C., y Palfai,T.(1995). Emotional attention, clarity, andrepair: Exploring emotional intelligence using theTrait Meta-Mood Scale. En J. W. Pennebaker (Ed.), Emotion, Disclosure and Health (pp. 125151). Washing-ton, United States of America: AmericanPsychological Association.

Sánchez, C., Ruiz, J., Campos,A., de laVega, M., Cortés, O., Esparza, M., Galbe, J., et al, (2019). Promoción de la actividad física en la infancia y la adolescencia (parte 1). Revista Pediatría Atención Primaria, 21, 79-91.

Santos, D. (2017). Estrategias para fortalecer la inteligencia emocional y favorecer el clima escolar en los niños de 5 a 6 años de la institución educativa Jorge Clemente Palacios de Tibasosa. Tesis doctoral, Universidad Santo Tomás.

Singh,H. (2017). Relationship between leisure-time physical activity and emotional intelligence in female university students: A correlational. European Journal of Physical Education and Sport Science, 3 (10), 209-216. https://doi.org/10.5281/ zenodo. 1001774.

Valenzuela, A., y Portillo, S. (2018). La inteligencia emocional en educación primaria y su relación con el rendimiento académico. Revista Electrónica Educare, 22(3), 1-15. https://doi.org/ 10.15359/ree.22-3.11

Vaquero, M., Amado, D., Sánchez, D., Sánchez, P., e Iglesias, D. (2020). Emotional Intelligence in Adolescence: Motivation and Physical Activity. Revista Internacional de Medicina y Ciencias de la Actividad Física y el Deporte, 20 (77), 119-131. https: / / doi.org/10.15366/rimcafd2020.77.008

Venkatappa, G., Shetty, S., Eregod, M., Girish, P., y Das, S. (2012). Gender differences in emotional intelligence among first year medical students. Journal of Evolution of medical and Dental Sciences, 1(6), 1256-1262. https://doi.org/10.14260/ jemds $/ 204$

Warburton, D., y Bredin, S. (2017). Health benefits of physical activity: A systematic review of current systematic reviews. Current Opinion in Cardiology, 32(5), 541-556.https: / / doi.org/ 10.1097/HCO.0000000000000437 